\title{
LATTICE RULES FOR MULTIPLE INTEGRATION AND DISCREPANCY
}

\author{
HARALD NIEDERREITER AND IAN H. SLOAN
}

\begin{abstract}
Upper and lower bounds for the discrepancy of nodes in lattice rules for multidimensional numerical integration are established. In this way the applicability of lattice rules is extended to nonperiodic integrands.
\end{abstract}

\section{INTRODUCTION}

Lattice rules for numerical integration over the $s$-dimensional unit cube $[0,1]^{s}$ were introduced by Sloan [11] and Sloan and Kachoyan [13], and the theory of lattice rules was developed further by Sloan and Walsh [12], Sloan and Kachoyan [14], and Sloan and Lyness [15]. An N-point lattice rule approximates the integral of a function $f$ over $[0,1]^{S}$ by

$$
\frac{1}{N} \sum_{n=0}^{N-1} f\left(\mathbf{x}_{n}\right)
$$

with distinct nodes $\mathbf{x}_{0}, \ldots, \mathbf{x}_{N-1} \in U^{s}=[0,1)^{s}$ for which the corresponding residue classes $\mathbf{x}_{0}+\mathbb{Z}^{s}, \ldots, \mathbf{x}_{N-1}+\mathbb{Z}^{s}$ form a subgroup $L$ of the torus group $\mathbb{R}^{s} / \mathbb{Z}^{s}$. Geometrically, this means that $L=\bigcup_{n=0}^{N-1}\left(\mathbf{x}_{n}+\mathbb{Z}^{s}\right)$, considered as a subset of $\mathbb{R}^{s}$, is a lattice in $\mathbb{R}^{s}$, whence the name "lattice rule". The special case where $L$ is a finite cyclic subgroup of $\mathbb{R}^{s} / \mathbb{Z}^{s}$ yields the number-theoretic method of good lattice points due to Korobov [5] and Hlawka [3] (see also [4, 8] for expository accounts of this method and the recent survey in [10]).

Lattice rules were originally conceived for the numerical integration of periodic functions having $[0,1]^{s}$ as their period interval, but the approximation (1) can of course also be used for nonperiodic integrands $f$. An upper bound for the integration error is obtained from the classical Koksma-Hlawka inequality [2] whenever the total variation $V(f)$ of $f$ over $[0,1]^{s}$ in the sense of Hardy and Krause is finite (compare also with [6, Chapter 2]). The resulting error bound is $V(f) D_{N}$, where $D_{N}$ is the discrepancy of the nodes $\mathbf{x}_{0}, \ldots, \mathbf{x}_{N-1}$. We recall that the discrepancy of any points $\mathbf{t}_{0}, \ldots, \mathbf{t}_{N-1} \in U^{S}$ is defined by

$$
D_{N}=D_{N}\left(\mathbf{t}_{0}, \ldots, \mathbf{t}_{N-1}\right)=\sup _{J}\left|\frac{A(J ; N)}{N}-\operatorname{Vol}(J)\right|,
$$

Received December 20, 1988.

1980 Mathematics Subject Classification (1985 Revision). Primary 65D32; Secondary 11H06, $11 \mathrm{~K} 38$. 
where $J$ runs through all half-open subintervals of $U^{s}$ of the form $J=$ $\prod_{j=1}^{s}\left[u_{j}, v_{j}\right), A(J ; N)$ is the number of integers $n, 0 \leq n \leq N-1$, with $\mathbf{t}_{n} \in J$, and $\operatorname{Vol}(J)$ denotes the volume of $J$.

In the present paper we study the discrepancy of the nodes in a lattice rule. We will establish upper and lower bounds for the discrepancy, which provide links with the figure of merit $\rho(L)$ introduced by Zaremba [16] for the method of good lattice points and extended to general lattice rules by Sloan and Kachoyan [14]. We will also point out some useful properties of $\rho(L)$. To define $\rho(L)$, we consider the dual lattice

$$
L^{\perp}=\left\{\mathbf{h} \in \mathbb{R}^{s}: \mathbf{h} \cdot \mathbf{x} \in \mathbb{Z} \text { for all } \mathbf{x} \in L\right\},
$$

where $\mathbf{h} \cdot \mathbf{x}$ denotes the standard inner product of $\mathbf{h}$ and $\mathbf{x}$. Since $L$ contains $\mathbb{Z}^{s}$ as a sublattice, it follows that $L^{\perp} \subseteq \mathbb{Z}^{s}$. For $h \in \mathbb{Z}$ put $r(h)=\max (1,|h|)$, and for $\mathbf{h}=\left(h_{1}, \ldots, h_{s}\right) \in \mathbb{Z}^{s}$ put

$$
r(\mathbf{h})=\prod_{j=1}^{s} r\left(h_{j}\right) .
$$

Then the figure of merit is defined by

$$
\rho(L)=\min _{\substack{\mathbf{h} \in L^{\perp} \\ \mathbf{h} \neq \mathbf{0}}} r(\mathbf{h}) .
$$

\section{UPPER BOUNDS FOR THE DISCREPANCY}

Let $\mathbf{x}_{0}, \ldots, \mathbf{x}_{N-1} \in U^{S}$ be the nodes in an $N$-point lattice rule with $N \geq 2$, and let $L$ be the corresponding lattice in $\mathbb{R}^{s}$. We assume from now on that $s \geq 2$, since the case $s=1$ is trivial (the nodes then form a set of equidistant points in $[0,1))$. Let $C(N)$ be the set of all nonzero $\mathbf{h}=\left(h_{1}, \ldots, h_{s}\right) \in \mathbb{Z}^{s}$ with $-N / 2<h_{j} \leq N / 2$ for $1 \leq j \leq s$. For integers $h \in(-N / 2, N / 2]$ we put

$$
r(h, N)= \begin{cases}N \sin (\pi|h| / N) & \text { if } h \neq 0 \\ 1 & \text { if } h=0\end{cases}
$$

For $\mathbf{h}=\left(h_{1}, \ldots, h_{s}\right) \in C(N)$ we write

$$
r(\mathbf{h}, N)=\prod_{j=1}^{s} r\left(h_{j}, N\right)
$$

Then we define

$$
R(L)=\sum_{\mathbf{h} \in\left(\left(\Lambda^{\prime}\right) \cap L^{+}\right.} \frac{1}{r(\mathbf{h}, N)}
$$

It is an easy result (see Proposition 3 in the next section) that $C(N) \cap L^{\perp}$ is nonempty for $s \geq 2$. 
Theorem 1. The discrepancy $D_{N}$ of the nodes $\mathbf{x}_{0}, \ldots, \mathbf{x}_{N-1} \in U^{s}$ in an $N$-point lattice rule satisfies

$$
D_{N} \leq \frac{s}{N}+R(L)
$$

Proof. By [14, Lemma 1] we have $N \mathbf{x}_{n} \in \mathbb{Z}^{s}$ for $0 \leq n \leq N-1$. Thus we can apply [7, Lemma 2.2], which yields

$$
D_{N} \leq \frac{s}{N}+\sum_{\mathbf{h} \in C(N)} \frac{1}{r(\mathbf{h}, N)}\left|\frac{1}{N} \sum_{n=0}^{N-1} e^{2 \pi i \mathbf{h} \cdot \mathbf{x}_{n}}\right| .
$$

For $\mathbf{h} \in \mathbb{Z}^{s}$ it was shown in [14, Theorem 1] that

$$
\frac{1}{N} \sum_{n=0}^{N-1} e^{2 \pi i \mathbf{h} \cdot \mathbf{x}_{n}}= \begin{cases}1 & \text { if } \mathbf{h} \in L^{\perp}, \\ 0 & \text { if } \mathbf{h} \notin L^{\perp},\end{cases}
$$

and so the desired result follows.

We show now that $R(L)$ can be bounded from above in terms of the figure of merit $\rho(L)$ defined in (2).

Theorem 2. We have

$$
R(L)<\frac{1}{\rho(L)}\left(\frac{2}{\log 2}\right)^{s-1}\left((\log N)^{s}+\frac{3}{2}(\log N)^{s-1}\right) .
$$

Proof. We have $r(h, N) \geq 2 r(h)$ for $0<|h| \leq N / 2$, since $\sin \pi x \geq 2 x$ for $0 \leq x \leq 1 / 2$, and also $r(0, N)=r(0)$. Thus, $r(\mathbf{h}, N) \geq 2 r(\mathbf{h})$ for all $\mathbf{h} \in C(N)$, and so

$$
R(L) \leq \frac{1}{2} \sum_{\mathbf{h} \in C(N) \cap L^{\perp}} \frac{1}{r(\mathbf{h})}=: \frac{1}{2} R_{1}(L) .
$$

To bound $R_{1}(L)$, we split up $C(N)$ into $2^{s}$ "quadrants" as follows. Put $I_{0}=(-N / 2,0], I_{1}=(0, N / 2]$, and for $\mathbf{d}=\left(d_{1}, \ldots, d_{s}\right)$ with $d_{j}=0$ or 1 , define

$$
Q(\mathbf{d})=\left\{\mathbf{h}=\left(h_{1}, \ldots, h_{s}\right) \in \mathbb{Z}^{s}: h_{j} \in I_{d}, \text { for } 1 \leq j \leq s \text { and } \mathbf{h} \neq \mathbf{0}\right\} .
$$

Then we can write

$$
R_{1}(L)=\sum_{\mathbf{d}} S(\mathbf{d}), \quad \text { with } S(\mathbf{d})=\sum_{\mathbf{h} \in Q(\mathbf{d}) \cap L^{\perp}} \frac{1}{r(\mathbf{h})} .
$$

We consider now $S(\mathbf{d})$ for fixed d . Let $\omega$ be the smallest integer with $2^{\omega}>$ $N / 2$, and let $\mu$ be the largest integer with $2^{\mu}<\rho(L)$; we can assume $\mu \geq 0$, since the case $\rho(L)=1$ is easily dealt with by [7, Lemma 2.3]. To allow us to further decompose the sum $S(\mathbf{d})$, we now define, for each $\mathbf{q}=\left(q_{2}, \ldots, q_{s}\right) \in$ $\mathbb{Z}^{s-1}$ with $1 \leq q_{j} \leq \omega, 2 \leq j \leq s$,

$$
M(\mathbf{q})=\left\{\left(h_{2}, \ldots, h_{s}\right) \in \mathbb{Z}^{s-1}: 2^{q_{j}-1} \leq r\left(h_{j}\right)<2^{q_{j}} \text { for } 2 \leq j \leq s\right\} .
$$


Then it is clear that we may write $S(\mathbf{d})$ as the sum, over all permissible vectors $\mathbf{q}$, of

$$
S(\mathbf{d}, \mathbf{q})=\sum_{\substack{\mathbf{h} \in Q(\mathbf{d}) \cap L^{\perp} \\\left(h_{2}, \ldots, h_{s}\right) \in M(\mathbf{q})}} \frac{1}{r(\mathbf{h})} .
$$

Case 1: $q_{2}+\cdots+q_{s} \leq \mu+s-1$. Put

$$
\Delta(\mathbf{q})=2^{-q_{2}-\cdots-q_{s}+s-1} \rho(L)>1,
$$

$K(\mathbf{q}, b)=\{h \in \mathbb{Z}: b \Delta(\mathbf{q}) \leq r(h)<(b+1) \Delta(\mathbf{q})\} \quad$ for $b \in \mathbb{Z}, 0 \leq b<[N / 2]$.

We claim that if $\mathbf{q}=\left(q_{2}, \ldots, q_{s}\right)$ belonging to Case 1 and $b \in \mathbb{Z}, 0 \leq b<$ $[N / 2]$, are given, then there exists at most one $\mathbf{h}=\left(h_{1}, \ldots, h_{s}\right) \in Q(\mathbf{d}) \cap$ $L^{\perp}$ such that $h_{1} \in K(\mathbf{q}, b)$ and $\left(h_{2}, \ldots, h_{s}\right) \in M(\mathbf{q})$. For suppose $\mathbf{h}^{\prime}=$ $\left(h_{1}^{\prime}, \ldots, h_{s}^{\prime}\right) \neq \mathbf{h}^{\prime \prime}=\left(h_{1}^{\prime \prime}, \ldots, h_{s}^{\prime \prime}\right)$ are two points satisfying all these conditions. Then $b \Delta(\mathbf{q}) \leq r\left(h_{1}^{\prime}\right), r\left(h_{1}^{\prime \prime}\right)<(b+1) \Delta(\mathbf{q})$ and $h_{1}^{\prime}, h_{1}^{\prime \prime} \in I_{d_{1}}$, hence $r\left(h_{1}^{\prime}-h_{1}^{\prime \prime}\right)<$ $\Delta(\mathbf{q})$. For $2 \leq j \leq s$ we have $2^{q_{j}-1} \leq r\left(h_{j}^{\prime}\right), r\left(h_{j}^{\prime \prime}\right)<2^{q_{j}}$ and $h_{j}^{\prime}, h_{j}^{\prime \prime} \in I_{d_{j}}$, hence $r\left(h_{j}^{\prime}-h_{j}^{\prime \prime}\right) \leq 2^{q_{j}-1}$. Therefore,

$$
r\left(\mathbf{h}^{\prime}-\mathbf{h}^{\prime \prime}\right)=\prod_{j=1}^{s} r\left(h_{j}^{\prime}-h_{j}^{\prime \prime}\right)<\Delta(\mathbf{q}) 2^{q_{2}+\cdots+q_{s}-s+1}=\rho(L) .
$$

On the other hand, $\mathbf{h}^{\prime}-\mathbf{h}^{\prime \prime} \in L^{\perp}$, since $L^{\perp}$ is an additive subgroup of $\mathbb{R}^{s}$, and also $\mathbf{h}^{\prime}-\mathbf{h}^{\prime \prime} \neq \mathbf{0}$, thus $r\left(\mathbf{h}^{\prime}-\mathbf{h}^{\prime \prime}\right) \geq \rho(L)$ by (2). This contradiction proves the claim.

Consider the contribution to $S(\mathbf{d}, \mathbf{q})$ arising from those $\mathbf{h}=\left(h_{1}, \ldots, h_{s}\right) \in$ $Q(\mathbf{d}) \cap L^{\perp}$ with $\left(h_{2}, \ldots, h_{s}\right) \in M(\mathbf{q})$ for which $h_{1} \in K(\mathbf{q}, b)$. For $b=0$ we have, trivially, $r(\mathbf{h}) \geq \rho(L)$, and for $b \geq 1$

$$
r(\mathbf{h}) \geq b \Delta(\mathbf{q}) 2^{q_{2}+\cdots+q_{s}-s+1}=b \rho(L) .
$$

Summing over $b$, we obtain

$$
S(\mathbf{d}, \mathbf{q}) \leq \frac{1}{\rho(L)}\left(1+\sum_{b=1}^{[N / 2]-1} \frac{1}{b}\right)<\frac{1}{\rho(L)}(1+\log N),
$$

where we used [7, Lemma 3.7].

Case 2: $q_{2}+\cdots+q_{s}>\mu+s-1$. Choose integers $\nu_{2}, \ldots, \nu_{s}$ with $0 \leq \nu_{j}<q_{j}$ for $2 \leq j \leq s$ and $\nu_{2}+\cdots+\nu_{s}=\mu$. For every $\mathbf{a}=\left(a_{2}, \ldots, a_{s}\right) \in \mathbb{Z}^{s-1}$ with $0 \leq a_{j}<2^{q_{j}-1,-1}$ for $2 \leq j \leq s$ let

$$
\begin{aligned}
M(\mathbf{q}, \mathbf{a})=\left\{\left(h_{2}, \ldots, h_{s}\right) \in \mathbb{Z}^{s-1}: 2^{q_{j}-1}\right. & +a_{j} 2^{\nu_{j}} \leq r\left(h_{j}\right) \\
& \left.<2^{q_{j}-1}+\left(a_{j}+1\right) 2^{\nu_{j}} \text { for } 2 \leq j \leq s\right\} .
\end{aligned}
$$

Then $M(\mathbf{q})$ defined above is the disjoint union of the sets $M(\mathbf{q}, \mathbf{a})$. Put

$$
K(b)=\{h \in \mathbb{Z}: 2 b \leq r(h) \leq 2 b+1\} \text { for } b \in \mathbb{Z}, 0 \leq b \leq[N / 4] .
$$


We claim that if $\mathbf{q}=\left(q_{2}, \ldots, q_{s}\right)$ belonging to Case $2, \mathbf{a}=\left(a_{2}, \ldots, a_{s}\right)$ satisfying the restrictions above, and $b \in \mathbb{Z}, 0 \leq b \leq[N / 4]$, are given, then there exists at most one $\mathbf{h}=\left(h_{1}, \ldots, h_{s}\right) \in Q(\mathbf{d}) \cap L^{\perp}$ such that $h_{1} \in K(b)$ and $\left(h_{2}, \ldots, h_{s}\right) \in M(\mathbf{q}, \mathbf{a})$. For suppose $\mathbf{h}^{\prime}=\left(h_{1}^{\prime}, \ldots, h_{s}^{\prime}\right) \neq \mathbf{h}^{\prime \prime}=\left(h_{1}^{\prime \prime}, \ldots, h_{s}^{\prime \prime}\right)$ are two points satisfying all these conditions. Then $h_{1}^{\prime}, h_{1}^{\prime \prime} \in K(b) \cap I_{d_{1}}$, hence $r\left(h_{1}^{\prime}-h_{1}^{\prime \prime}\right)=1$, and for $2 \leq j \leq s$ we have $r\left(h_{j}^{\prime}-h_{j}^{\prime \prime}\right) \leq 2^{\nu_{j}}$. Therefore,

$$
r\left(\mathbf{h}^{\prime}-\mathbf{h}^{\prime \prime}\right)=\prod_{j=1}^{s} r\left(h_{j}^{\prime}-h_{j}^{\prime \prime}\right) \leq 2^{\nu_{2}+\cdots+\nu_{s}}=2^{\mu}<\rho(L) .
$$

On the other hand, $\mathbf{h}^{\prime}-\mathbf{h}^{\prime \prime} \in L^{\perp}$ and $\mathbf{h}^{\prime}-\mathbf{h}^{\prime \prime} \neq \mathbf{0}$, thus $r\left(\mathbf{h}^{\prime}-\mathbf{h}^{\prime \prime}\right) \geq \rho(L)$ by (2). This contradiction proves the claim.

Since there are $\prod_{j=2}^{s} 2^{q_{j}-\nu_{j}-1}=2^{q_{2}+\cdots+q_{s}-\mu-s+1}$ choices for a, it follows that if $b$ is given as above, then there are at most $2^{q_{2}+\cdots+q_{s}-\mu-s+1}$ points $\mathbf{h}=$ $\left(h_{1}, \ldots, h_{s}\right) \in Q(\mathbf{d}) \cap L^{\perp}$ such that $h_{1} \in K(b)$ and $\left(h_{2}, \ldots, h_{s}\right) \in M(\mathbf{q})$. Therefore,

$$
\begin{aligned}
S(\mathbf{d}, \mathbf{q}) & \leq 2^{q_{2}+\cdots+q_{s}-\mu-s+1} 2^{-q_{2}-\cdots-q_{s}+s-1}\left(1+\sum_{b=1}^{[N / 4]} \frac{1}{2 b}\right) \\
& =2^{-\mu}\left(1+\frac{1}{2} \sum_{b=1}^{[N / 4]} \frac{1}{b}\right) \leq \frac{1}{\rho(L)}\left(2+\sum_{b=1}^{[N / 4]} \frac{1}{b}\right),
\end{aligned}
$$

where we used $2^{\mu+1} \geq \rho(L)$ in the last step. Applying [7, Lemma 3.7] with $m=[N / 2]+2$, we get

$$
\sum_{b=1}^{[N / 4]} \frac{1}{b}<\log \left(\left[\frac{N}{2}\right]+2\right)
$$

and so for $N \geq 19$,

$$
\sum_{b=1}^{[N / 4]} \frac{1}{b}<\log N-\frac{1}{2}
$$

The last inequality is shown by inspection for $2 \leq N \leq 18, N \neq 4$. Thus for $N \neq 4$,

$$
S(\mathbf{d}, \mathbf{q})<\frac{1}{\rho(L)}\left(\frac{3}{2}+\log N\right) .
$$

By (5) this bound can also be used in Case 1. Since there are $\omega^{s-1}$ choices for $\mathbf{q}$, we get

$$
S(\mathbf{d})<\frac{1}{\rho(L)} \omega^{s-1}\left(\frac{3}{2}+\log N\right) .
$$

There are $2^{s}$ possibilities for $\mathbf{d}$, hence

$$
R_{1}(L)<\frac{1}{\rho(L)}(2 \omega)^{s-1}(3+2 \log N) .
$$


Since the definition of $\omega$ implies $2^{(1)} \leq N$, we obtain

$$
R_{1}(L)<\frac{1}{\rho(L)}\left(\frac{2}{\log 2}\right)^{s-1}\left(2(\log N)^{s}+3(\log N)^{s-1}\right),
$$

and together with (4) this completes the proof for $N \neq 4$. For $N=4$ we trivially have

$$
R(L) \leq \sum_{\mathbf{h} \in C^{(4)}} \frac{1}{r(\mathbf{h}, 4)}<\left(\sum_{h=-1}^{2} \frac{1}{r(h, 4)}\right)^{s}<2^{s},
$$

and since $\rho(L) \leq 4$ by Proposition 2 below, the inequality of Theorem 2 is checked immediately for $s \geq 3$. In the remaining case $N=4$ and $s=2$, we note that $r(\mathbf{h})=1,2,4$ for $\mathbf{h} \in C(4)$, and so by Proposition 1 below, $\rho(L)$ can only attain the values $1,2,4$. If $\rho(L) \leq 2$, then $R(L)<4$ shows that the inequality of Theorem 2 holds. If $\rho(L)=4$, then $C(4) \cap L^{\perp}$ consists only of the point $(2,2)$, which is impossible, since it contradicts Proposition 3 below.

By combining Theorems 1 and 2 we obtain the discrepancy bound

$$
D_{N}<\frac{s}{N}+\frac{1}{\rho(L)}\left(\frac{2}{\log 2}\right)^{s-1}\left((\log N)^{s}+\frac{3}{2}(\log N)^{s-1}\right) .
$$

Since $\rho(L) \leq N$ by Proposition 2 below, it follows that $D_{N}=O\left(\rho(L)^{-1}(\log N)^{s}\right)$ with an implied constant depending only on the dimension $S$.

Remark 1. A similar discrepancy bound can also be obtained in the more general case of a displaced lattice rule. According to [14, p. 119], if $\mathbf{x}_{0}, \ldots, \mathbf{x}_{N-1}$ are the nodes in a lattice rule, then the nodes in a corresponding displaced lattice rule are given by $\left\{\mathbf{x}_{0}+\mathbf{y}\right\}, \ldots,\left\{\mathbf{x}_{N-1}+\mathbf{y}\right\}$ with $\mathbf{y} \in U^{S}$, where the fractional part $\{\mathbf{t}\} \in U^{s}$ is obtained by reducing all coordinates of $\mathbf{t} \in \mathbb{R}^{s}$ modulo 1. We can write $\mathbf{y}=\mathbf{y}^{\prime}+\mathbf{z}$, where $N \mathbf{y}^{\prime} \in \mathbb{Z}^{s}$ and $\mathbf{z} \in[0,1 / N)^{s}$. The method in the proof of Theorem 1 shows that the discrepancy $D_{N}^{\prime}$ of the points $\left\{\mathbf{x}_{0}+\mathbf{y}^{\prime}\right\}, \ldots,\left\{\mathbf{x}_{N-1}+\mathbf{y}^{\prime}\right\}$ satisfies

$$
D_{N}^{\prime} \leq \frac{s}{N}+R(L)
$$

where $L$ is the lattice $\bigcup_{n=0}^{N-1}\left(\mathbf{x}_{n}+\mathbb{Z}^{s}\right)$. Since $\left\{\mathbf{x}_{n}+\mathbf{y}\right\}=\left\{\mathbf{x}_{n}+\mathbf{y}^{\prime}\right\}+\mathbf{z}$ for $0 \leq n \leq$ $N-1$, it is easily seen (compare with the argument in the proof of $[6$, p. 132, Theorem 4.1]) that the discrepancy $D_{N}$ of the points $\left\{\mathbf{x}_{0}+\mathbf{y}\right\}, \ldots,\left\{\mathbf{x}_{N-1}+\mathbf{y}\right\}$ satisfies

$$
D_{N} \leq \frac{s}{N}+D_{N}^{\prime} \leq \frac{2 s}{N}+R(L) \text {. }
$$

Together with Theorem 2 we get a discrepancy bound analogous to (6).

\section{Properties of THE Figure of Merit}

We prove simple properties of the figure of merit $\rho(L)$, already used in $\S 2$, and also of general interest in the theory of lattice rules. 
Proposition 1. The figure of merit $\rho(L)$ is also given by

$$
\rho(L)=\min _{\mathbf{h} \in C(N) \cap L^{\perp}} r(\mathbf{h}) .
$$

Proof. By [14, Lemma 1] the nodes $\mathbf{x}_{0}, \ldots, \mathbf{x}_{N-1}$ of the lattice rule satisfy $N \mathbf{x}_{n} \in \mathbb{Z}^{s}$ for $0 \leq n \leq N-1$, hence $L^{\perp}$ contains $(N \mathbb{Z})^{s}$ as a subgroup. Since $L^{\perp}$ has determinant $N$ by [14, pp. 119-120], and the cube $\left[-N^{1 / s}, N^{1 / s}\right]^{s}$ has volume $2^{s} N$, it follows from Minkowski's convex body theorem [1, p. 71] that this cube contains an element $\mathbf{h}_{0} \neq \mathbf{0}$ of $L^{\perp}$. Clearly, $r\left(\mathbf{h}_{0}\right) \leq N$. Since $\mathbf{h}_{0} \in \mathbb{Z}^{s}$ and $\left[N^{1 / s}\right] \leq N / 2$ for $N \geq 2$ and $s \geq 2$, we have $\mathbf{h}_{0} \in[-N / 2, N / 2]^{s}$. By replacing, if necessary, every coordinate of $\mathbf{h}_{0}$ equal to $-N / 2$ by $N / 2$, we get an $\mathbf{h}_{1} \in C(N) \cap L^{\perp}$ with $r\left(\mathbf{h}_{1}\right) \leq N$. To prove the proposition, it suffices to show that for any $\mathbf{h} \in L^{\perp}, \mathbf{h} \neq \mathbf{0}$, there exists an $\mathbf{h}_{2} \in C(N) \cap L^{\perp}$ with $r\left(\mathbf{h}_{2}\right) \leq r(\mathbf{h})$. If all coordinates of $\mathbf{h}$ are multiples of $N$, then $r(\mathbf{h}) \geq N$, and so we can take $h_{2}=h_{1}$. Otherwise, we obtain a suitable $h_{2}$ by reducing each coordinate of $\mathbf{h}$ modulo $N$ to get numbers in $(-N / 2, N / 2]$.

Proposition 2. We always have $\rho(L) \leq N$.

Proof. Since $L^{\perp}$ contains $(N \mathbb{Z})^{s}$, we have $\mathbf{h}=(N, 0, \ldots, 0) \in L^{\perp}$, hence $\rho(L) \leq r(\mathbf{h})=N$.

Proposition 3. The set $C(N) \cap L^{\perp}$ contains exactly $N^{s-1}-1$ elements.

Proof. Let $\mathbf{z} \in \mathbb{Z}^{s}$, and consider the translate $N \mathbf{z}+C^{*}(N)$, where $C^{*}(N)=$ $C(N) \cup\{\boldsymbol{0}\}$. Since $L^{\perp}$ contains $(N \mathbb{Z})^{s}$, it is clear that $\left(N \mathbf{z}+C^{*}(N)\right) \cap L^{\perp}=$ $N \mathbf{z}+\left(C^{*}(N) \cap L^{\perp}\right)$, so that $\left(N \mathbf{z}+C^{*}(N)\right) \cap L^{\perp}$ contains the same number of elements as $C^{*}(N) \cap L^{\perp}$, say $\nu(N)$. Since $L^{\perp}$ is the union of disjoint subsets $\left(N \mathbf{z}+C^{*}(N)\right) \cap L^{\perp}$, each containing $\nu(N)$ elements, that number is obtained by dividing the volume of the cube $(-N / 2, N / 2]^{s}$, namely $N^{s}$, by the determinant of $L^{\perp}$, which by [14, pp. 119-120] has the value $N$. Thus $\nu(N)=N^{s-1}$. Finally, $C(N) \cap L^{\perp}$ contains $\nu(N)-1$ elements, because it does not contain zero.

\section{LOWER BOUNDS FOR THE DISCREPANCY}

Let $D_{N}$ again be the discrepancy of the nodes $\mathbf{x}_{0}, \ldots, \mathbf{x}_{N-1} \in U^{s}$ in an $N$ point lattice rule. A simple lower bound for $D_{N}$ is obtained as follows. Since $N \mathbf{x}_{n} \in \mathbb{Z}^{s}$ for $0 \leq n \leq N-1$, we have $\mathbf{x}_{n} \in[0,1-1 / N]^{s}$ for $0 \leq n \leq N-1$. For $0<\varepsilon \leq 1 / N$ let $J_{\varepsilon}=[0,1-1 / N+\varepsilon)^{s}$. Then the definition of $D_{N}$ implies

$$
D_{N} \geq\left|\frac{A\left(J_{\varepsilon} ; N\right)}{N}-\operatorname{Vol}\left(J_{\varepsilon}\right)\right|=1-\left(1-\frac{1}{N}+\varepsilon\right)^{s} .
$$

Letting $\varepsilon \rightarrow 0+$, we obtain

$$
D_{N} \geq 1-\left(1-\frac{1}{N}\right)^{s} .
$$


In first approximation this lower bound is equal to $s / N$, which happens to be the first term in the upper bound (6).

A more important result is the lower bound for $D_{N}$ in terms of the figure of merit $\rho(L)$ to be shown in Theorem 3. We need an auxiliary result which is a variant of [9, Lemma 5.4].

Lemma. For any points $\mathbf{t}_{0}, \ldots, \mathbf{t}_{N-1} \in U^{s}$, any nonzero $\mathbf{h} \in \mathbb{Z}^{s}$, and any real $\theta$ we have

$$
\left|\frac{1}{N} \sum_{n=0}^{N-1} \cos 2 \pi\left(\mathbf{h} \cdot \mathbf{t}_{n}-\theta\right)\right| \leq \frac{2}{\pi}\left((\pi+1)^{s}-1\right) r(\mathbf{h}) D_{N}\left(\mathbf{t}_{0}, \ldots, \mathbf{t}_{N-1}\right) .
$$

Proof. By $[9$, p. 64] we have

$$
\left|\frac{1}{N} \sum_{n=0}^{N-1} \cos 2 \pi\left(\mathbf{h} \cdot \mathbf{t}_{n}-\theta\right)\right| \leq \frac{2}{\pi}\left((\pi+1)^{s}-1\right)\left|h_{1} \cdots h_{s}\right| D_{N}\left(\mathbf{t}_{0}, \ldots, \mathbf{t}_{N-1}\right)
$$

provided that all coordinates $h_{1}, \ldots, h_{s}$ of $\mathbf{h}$ are $\neq 0$. Since then $r(\mathbf{h})=$ $\left|h_{1} \cdots h_{s}\right|$, the lemma holds in this case. Now we take an arbitrary $\mathbf{h}=$ $\left(h_{1}, \ldots, h_{s}\right) \neq \mathbf{0}$ and we assume without loss of generality that $h_{j} \neq 0$ for $1 \leq j \leq k$ and $h_{j}=0$ for $k+1 \leq j \leq s$. Let $\mathbf{h}^{\prime}=\left(h_{1}, \ldots, h_{k}\right)$, and if $\mathbf{t}_{n}=\left(t_{n}^{(1)}, \ldots, t_{n}^{(s)}\right)$, we put $\mathbf{t}_{n}^{\prime}=\left(t_{n}^{(1)}, \ldots, t_{n}^{(k)}\right)$. By what we have already shown, we get

$$
\begin{aligned}
\left|\frac{1}{N} \sum_{n=0}^{N-1} \cos 2 \pi\left(\mathbf{h} \cdot \mathbf{t}_{n}-\theta\right)\right| & =\left|\frac{1}{N} \sum_{n=0}^{N-1} \cos 2 \pi\left(\mathbf{h}^{\prime} \cdot \mathbf{t}_{n}^{\prime}-\theta\right)\right| \\
& \leq \frac{2}{\pi}\left((\pi+1)^{k}-1\right) r\left(\mathbf{h}^{\prime}\right) D_{N}\left(\mathbf{t}_{0}^{\prime}, \ldots, \mathbf{t}_{N-1}^{\prime}\right) \\
& \leq \frac{2}{\pi}\left((\pi+1)^{s}-1\right) r\left(\mathbf{h}^{\prime}\right) D_{N}\left(\mathbf{t}_{0}^{\prime}, \ldots, \mathbf{t}_{N-1}^{\prime}\right) .
\end{aligned}
$$

Since $r\left(\mathbf{h}^{\prime}\right)=r(\mathbf{h})$ and

$$
D_{N}\left(\mathbf{t}_{0}^{\prime}, \ldots, \mathbf{t}_{N-1}^{\prime}\right) \leq D_{N}\left(\mathbf{t}_{0}, \ldots, \mathbf{t}_{N-1}\right),
$$

we have proved the lemma in the general case.

Theorem 3. The discrepancy $D_{N}$ of the nodes $\mathbf{x}_{0}, \ldots, \mathbf{x}_{N-1} \in U^{S}$ in an $N$-point lattice rule satisfies

$$
D_{N^{\prime}} \geq \frac{1}{C_{s} \rho(L)}
$$

with $C_{2}=4, C_{3}=27$, and $C_{s}=\frac{2}{\pi}\left((\pi+1)^{s}-1\right)$ for $s \geq 4$.

Proof. Choose $\mathbf{h} \in L^{\perp}, \mathbf{h} \neq \mathbf{0}$, with $r(\mathbf{h})=\rho(L)$. Then $\mathbf{h} \cdot \mathbf{x}_{n} \in \mathbb{Z}$ for $0 \leq$ $n \leq N-1$, and so an application of the lemma with $\mathbf{t}_{n}=\mathbf{x}_{n}$ for $0 \leq n \leq N-1$ and $\theta=0$ yields

$$
1 \leq \frac{2}{\pi}\left((\pi+1)^{s}-1\right) \rho(L) D_{N} .
$$

This is the desired result for $s \geq 4$. For $s=2$ and $s=3$ we use a different method to get a smaller value of $C_{s}$. Choose $\mathbf{h}=\left(h_{1}, \ldots, h_{s}\right)$ as before and 
assume first that $\sum_{j=1}^{s}\left|h_{j}\right| \geq 2$. Then an application of [9, Lemma 5.5] with $\mathbf{t}_{n}=\mathbf{x}_{n}$ for $0 \leq n \leq N-1$ and $\theta=0$ yields

$$
D_{N} \geq \frac{1}{s^{s} \rho(L)} .
$$

If $\sum_{j=1}^{s}\left|h_{j}\right|=1$, then for some $i, 1 \leq i \leq s$, we have $h_{i}= \pm 1$ and $h_{j}=0$ for all $j \neq i$. From $\mathbf{h} \cdot \mathbf{x}_{n} \in \mathbb{Z}$ it follows then that the $i$ th coordinate of each $\mathbf{x}_{n}$ is 0 . This yields $D_{N}=1$, and so (7) holds trivially. From (7) we get the desired value of $C_{s}$ for $s=2$ and $s=3$.

Remark 2. The lower bound in Theorem 3 holds also for the nodes $\mathbf{y}_{n}=\left\{\mathbf{x}_{n}+\right.$ $\mathbf{y}\}, 0 \leq n \leq N-1$, in a displaced lattice rule (compare with Remark 1). In the first part of the proof of Theorem 3 one applies the lemma with $\mathbf{t}_{n}=\mathbf{y}_{n}$ for $0 \leq n \leq N-1$ and $\theta=\mathbf{h} \cdot \mathbf{y}$. In the second part (cases $s=2$ and $s=3$ ) one applies [9, Lemma 5.5] with $\mathbf{t}_{n}=\mathbf{y}_{n}$ for $0 \leq n \leq N-1$ and $\theta=\{\mathbf{h} \cdot \mathbf{y}\}$, and this yields (7) provided that $\sum_{j=1}^{s}\left|h_{j}\right| \geq 2$. If $\sum_{j=1}^{s}\left|h_{j}\right|=1$, then for some $i$, $1 \leq i \leq s$, each $\mathbf{y}_{n}$ has the same $i$ th coordinate, and this implies $D_{N} \geq \frac{1}{2}$, so that (7) holds again.

\section{Conclusions}

It follows from the upper bound in (6) and the lower bound in Theorem 3 that these two results are best possible up to factors of the order $(\log N)^{s}$. Furthermore, these two results show that the order of magnitude of the discrepancy of nodes in a lattice rule is essentially given by $1 / \rho(L)$ (compare also with the information on $\rho(L)$ given in the next paragraph). This suggests that the figure of merit $\rho(L)$ of the lattice $L$ should be large if one wants to obtain an efficient numerical integration method, agreeing with the conclusion reached by Sloan and Kachoyan [14] in their analysis of lattice rules with periodic integrands. Similar comments apply to displaced lattice rules because of Remarks 1 and 2 .

The simple upper bound for $\rho(L)$ in Proposition 2 is nearly best possible. In fact, in the special case of the method of good lattice points one can already find, for each $s \geq 2$ and all sufficiently large $N$, a suitable lattice $L$ such that $\rho(L)$ is at least of the order of magnitude $N /(\log N)^{s-1}$, and in the case $s=2$ there exist infinitely many $N$ and corresponding lattices $L$ such that $\rho(L)$ is of the order of magnitude $N$ (see $[8, \S 4]$ ). Therefore, $N$-point lattice rules are capable of producing error bounds of the form $O\left(N^{-1}(\log N)^{c(s)}\right)$ when applied to integrands of bounded variation in the sense of Hardy and Krause, where the constant $c(s)>0$ depends only on $s$. An important task that remains to be carried out is to find many concrete examples of lattice rules that improve on the method of good lattice points, e.g., in the sense of a larger figure of merit.

\section{BIBLIOGRAPHY}

1. J. W. S. Cassels, An introduction to the geometry of numbers, Springer, Berlin, 1971.

2. E. Hlawka, Funktionen von neschränkter Variation in der Theorie der Gleichverteilung. Ann. Mat. Pura Appl. 54 (1961), 325-333.

3. $\frac{1}{151}$. Zur angenäherten Berechnung mehrfacher Integrale, Monatsh. Math. 66 (1962), 140151. 
4. L. K. Hua and Y. Wang, Applications of number theory to numerical analysis, Springer, Berlin, 1981.

5. N. M. Korobov, The approximate computation of multiple integrals, Dokl. Akad. Nauk SSSR 124 (1959), 1207-1210. (Russian)

6. L. Kuipers and H. Niederreiter, Lniform distribution of sequences, Wiley, New York, 1974.

7. H. Niederreiter, Pseudo-random numbers and optimal coefficients, Adv. in Math. 26 (1977), 99-181.

8. __ Quasi-Monte Carlo methods and pseudo-random numbers, Bull. Amer. Math. Soc. 84 (1978), 957-1041.

9. ___ The serial test for pseudo-random numbers generated by the linear congruential method, Numer. Math. 46 (1985), 51-68.

10. __, Quasi-Monte Carlo methods for multidimensional numerical integration, Numerical Integration (Oberwolfach, 1987) (H. Brass and G. Hämmerlin, eds.), ISNM 85, Birkhäuser, Basel, 1988, pp. 157-171.

11. I. H. Sloan, Lattice methods for multiple integration, J. Comput. Appl. Math. 12/13 (1985), 131-143.

12. I. H. Sloan and L. Walsh, Lattice rules-classification and searches, Numerical Integration (Oberwolfach, 1987) (H. Brass and G. Hämmerlin, eds.), ISNM 85, Birkhäuser, Basel, 1988, pp. 251-260.

13. I. H. Sloan and P. J. Kachoyan, Lattice methods for multiple integration, Proc. Centre Math. Anal. Austral. Nat. Univ., vol. 6, Austral. Nat. Univ., Canberra, 1984, pp. 147-165.

14. __ Lattice methods for multiple integration: theory, error analysis and examples, SIAM J. Numer. Anal. 24 (1987), 116-128.

15. I. H. Sloan and J. N. Lyness, The representation of lattice quadrature rules as multiple sums, Math. Comp. 52 (1989), 81-94.

16. S. K. Zaremba, La méthode des 'bons treillis' pour le calcul des intégrales multiples, in Applications of Number Theory to Numerical Analysis (S. K. Zaremba, ed.), Academic Press, London, 1972, pp. 39-116.

Institute for Information Processing, Austrian Academy of Sciences, Dr.-IgnazSeipel-Platz 2, A-1010 Vienna, Austria

School of Mathematics, University of New South Wales, Sydney, New South Wales 2033, Australia 